\title{
TESCAN Cryo FIB-SEM as a Flexible Tool for Advanced Sample Analysis
}

Rostislav Váňa ${ }^{1}$, Kristýna Rosíková ${ }^{2}$, Jakub Javůrek ${ }^{2}$, Tomáš Nováček ${ }^{1}$, Jana Havránková ${ }^{2}$ and Samuel Záchej ${ }^{1}$

1. TESCAN Brno, Brno, Czech Republic.

2. TESCAN ORSAY HOLDING, Brno, Czech Republic.

Low temperature scanning electron microscopy (SEM) has become an established technique for an analysis of beam-sensitive materials and hydrated biological samples close to their natural state. The Cryo FIB-SEM technique is widely used for a broad range of applications in Life sciences, especially for soft biological specimens, and for materials such as polymers, gels, foams or emulsions. TESCAN instruments can be equipped with a variety of cryo-processing tools. This allows researchers to take advantage of all TESCAN SEM and FIB-SEM capabilities with the state-of-the-art cryo-technology of their choice.

TESCAN FIB-SEM workstations serve as an advanced sample preparation tool for ultra-high resolution TEM analysis. FIB fabrication is used extensively in material sciences and semiconductor industry either to reveal hidden information from subsurface layers by cross-sectioning or to prepare ultra-thin lamellae from bulk materials for subsequent transmission electron microscopy (TEM). However, soft materials suffer from a serious heat damage during the thinning phase, which can be caused by a heat transfer from the ion beam. When the sample is cooled to the cryogenic temperature during the final thinning, the effect of melting is dramatically decreased.

The technique of the thin lamellae preparation is becoming increasingly popular also in structural biology, where the method of choice is cryo-electron tomography (cryo-ET). FIB processing of thick plunge-frozen eukaryotic cells or other inaccessible samples for the TEM, which comprises site-specific navigation, deposition of protection layer and FIB thinning, delivers artefact-free lamellae in a preserved state. An example of the thin lamella preparation from an eukaryotic cell is on Fig. 1.

TESCAN integration of analytical modalities such as Time of Flight (ToF) SIMS and Energy Dispersive X-Ray Spectroscopy (EDS) to the FIB-SEM provides additional information on elemental sample composition even under the cryogenic conditions. An example of the cryo-ToF application for analysis of the soft material is on Fig. 2. The spectra was acquired from a hand cream, which contains $\mathrm{Ca}$ aggregates. The analytical power of the combined system might be useful for analysis of various liquids, reactive or sensitive materials in the pharmaceutical, food or material industry.

The aim of this presentation is to demonstrate the flexibility of TESCAN FIB-SEM system on various challenging cryo-applications, such as fabrication of thin cryo-TEM lamellas, cross-sectioning and chemical mapping of beam sensitive samples. 


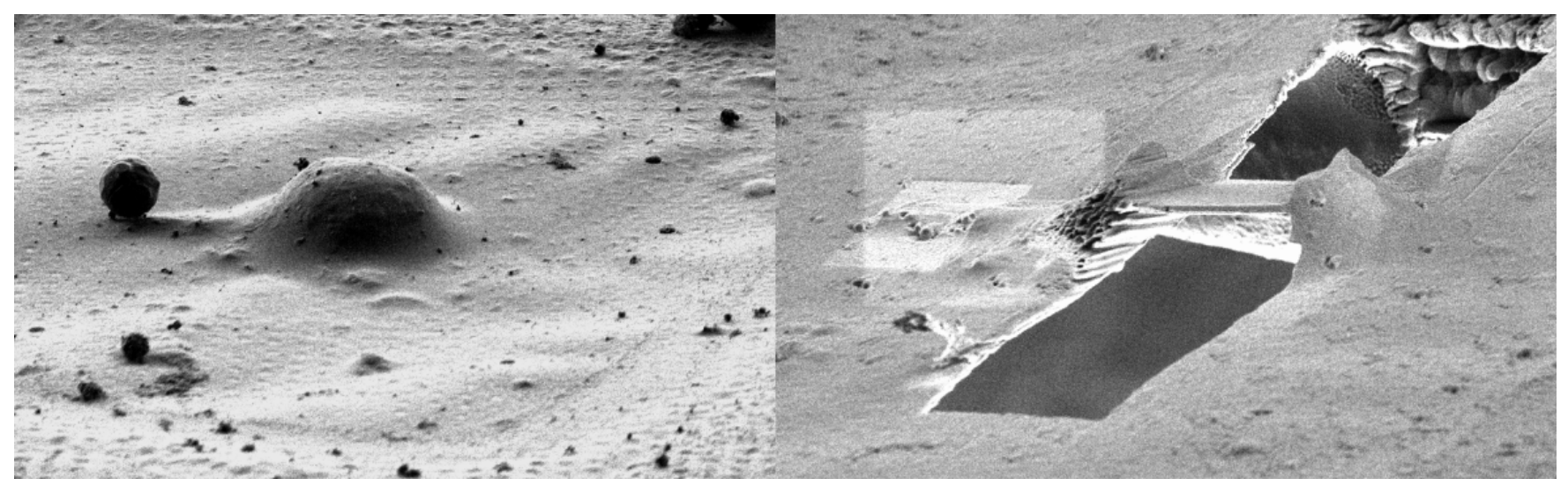

Figure 1. TEM lamella prepared from a frozen cell using the TESCAN S8000G Cryo-FIB. Top left - selected ROI of a single cell on the TEM grid; top right - a thin lamella fabricated from the selected cell. Instrument used - TESCAN S8000G.
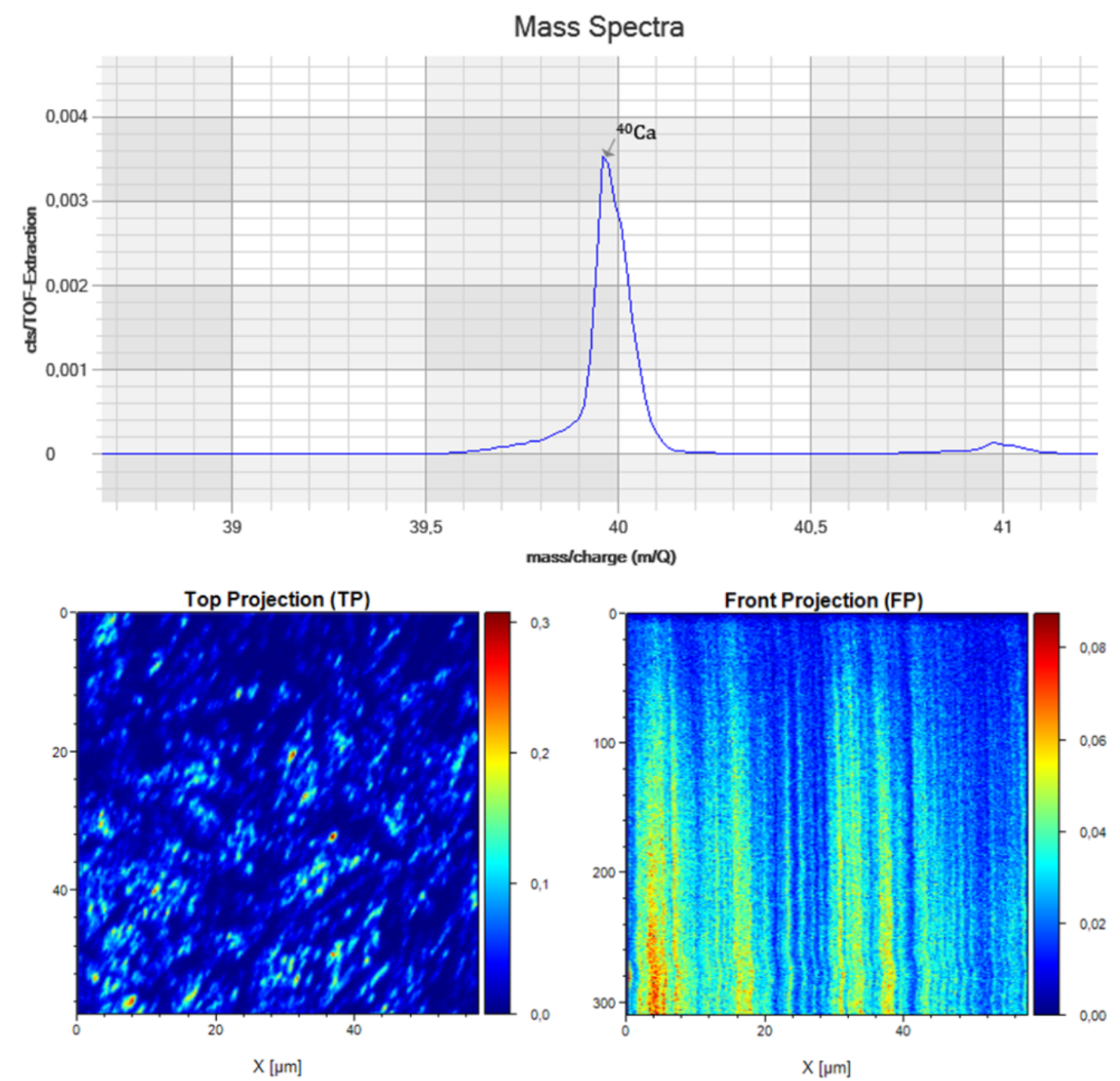

Figure 2. Analysis of a cosmetic cream containing $\mathrm{Ca}$ additives. Top - acquired mass spectrum with a significant peak of Ca. The exact location of the Ca regions can be observed on a Top Projection (Bottom left) or a depth distribution using a Front Projection image (Bottom right). ToF SIMS data can be directly correlated with an appropriate SEM micrograph to combine the detailed structural and compositional information. 